\title{
Correction to: Histone demethylase JMJD2D activates HIF1 signaling pathway via multiple mechanisms to promote colorectal cancer glycolysis and progression
}

\author{
Kesong Peng · Minghui Zhuo · Ming Li - Qiang Chen · Pingli Mo • Chundong Yu
}

Published online: 22 February 2021

(c) The Author(s), under exclusive licence to Springer Nature Limited 2021

Correction to: Oncogene

https://doi.org/10.1038/s41388-020-01483-w

The original version of this article contained an error in Fig. 8a. An incorrect image was shown for the correlation between the expression of JMJD2D and MCT4. Accordingly, the sentence "The mRNA levels of JMJD2D were positively correlated with mTOR, HIF1 $\beta$, PGK1, and LDHA in 48-paired human CRC specimens." in the figure caption of Fig. 8 was replaced by "The mRNA levels of
JMJD2D were positively correlated with mTOR, HIF1 $\beta$, PGK1, and LDHA in 48-paired human CRC specimens.", and the sentence "As shown in Fig. 8a, the mRNA expression of JMJD2D was positively correlated with mTOR, HIF1 $\beta$, PGK1, LDHA, and MCT4." in the last part of the results section was replaced by "As shown in Fig. 8a, the mRNA expression of JMJD2D was positively correlated with mTOR, HIF1 $\beta$, PGK1 and LDHA, but not MCT4.". The corrected figure and figure caption are given below. The original article has been corrected. 
a
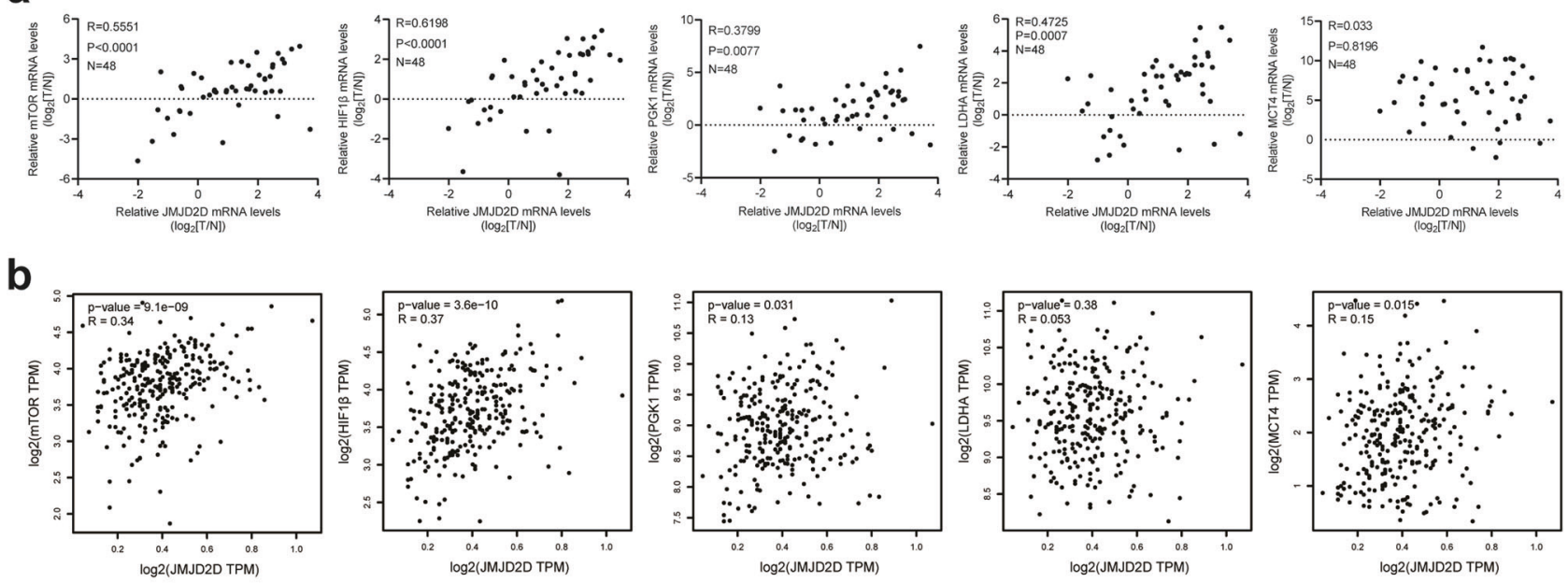

C

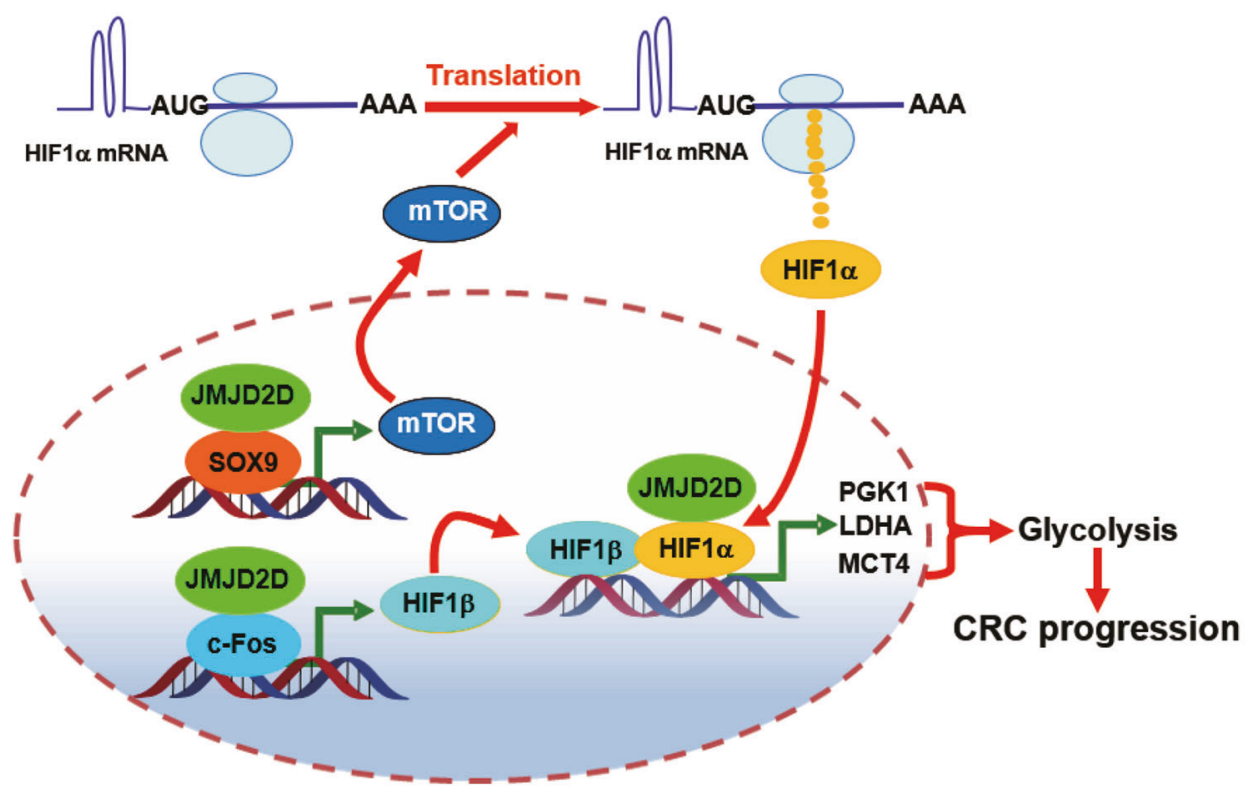

CRC cell

Fig. 8 The mRNA levels of JMJD2D is positively correlated with mTOR, HIF1及, and glycolytic genes in human CRC specimens. a The mRNA levels of JMJD2D were positively correlated with mTOR, HIF1 $\beta$, PGK1, and LDHA in 48-paired human CRC specimens. b The mRNA levels of JMJD2D were positively correlated with mTOR, HIF1 $\beta$, PGK1, and MCT4 in TCGA database. c Schematic model for the mechanisms by which JMJD2D activates HIF1 signaling to promote CRC cell glycolysis and progression. 